\section{RMD Open}

Rheumatic \&

Musculoskeletal Diseases

\title{
Estimation of treatment and prognostic factors of pneumocystis pneumonia in patients with connective tissue diseases
}

To cite: Ishikawa Y, Nakano K, Tokutsu K, et al. Estimation of treatment and prognostic factors of pneumocystis pneumonia in patients with connective tissue diseases. RMD Open 2021;7:e001508. doi:10.1136/ rmdopen-2020-001508

Received 6 November 2020 Revised 5 February 2021 Accepted 22 February 2021

\section{Check for updates}

(c) Author(s) (or their employer(s)) 2021. Re-use permitted under CC BY-NC. No commercial re-use. See rights and permissions. Published by BMJ.

${ }^{1}$ The First Department of Internal Medicine, University of Occupational and Environmental Health, Japan, Kitakyushu, Japan

'Department of Rheumatology, Yokohama Rosai Hospital, Yokohama, Japan

${ }^{3}$ Sato Clinic, Tokyo, Japan

${ }^{4}$ Department of Preventive

Medicine and Community Health, University of Occupational and Environmental Health Japan, Kitakyushu, Fukuoka, Japan ${ }^{5}$ Department of Environmental Epidemiology, University of Occupational and Environmental Health Japan, Kitakyushu, Japan

Correspondence to Professor Yoshiya Tanaka; tanaka@med.uoeh-u.ac.jp

\section{ABSTRACT}

Objectives To investigate short-term prognosis and prognostic factors for connective tissue disease-associated pneumocystis pneumonia (CTD-PCP) using the Japanese nationwide diagnosis procedure combination (DPC) inpatient database.

Methods The present retrospective cohort study from April 2014 to March 2016 included data of patients with CTD-PCP extracted from the DPC database using the $10^{\text {th }}$ revision of International Classification of Diseases and Injuries codes.

Results In 15901766 cases registered from 1329 hospitals, 333 of 67890 patients who were admitted with PCP were diagnosed with CTD-PCP and included in the study. The median age was 71.0 years, and 214 (64.3\%), 80 (24.0\%), and 29 (8.7\%) patients received sulfamethoxazole/trimethoprim (ST) monotherapy and pentamidine-containing and atovaquone-containing therapy, respectively. There were 114 (34.2\%) in-hospital deaths, and the 30-day and 60-day in-hospital survival rates after PCP treatment initiation were $66.0 \%$ and $53.7 \%$, respectively. Older age (HR $1.06,95 \% \mathrm{Cl} 1.03$ to 1.08) and concomitant interstitial lung disease (ILD) (HR $1.65,95 \% \mathrm{Cl} 1.12$ to 2.42) were poor prognostic factors. Patients who completed PCP treatment with ST monotherapy had a significantly higher survival rate than those treated with those not treated with ST monotherapy $(p=0.015$; log-rank test). Pentamidine versus atovaquone as second-line therapy was significantly higher with atovaquone ( $p=0.012$; log-rank test).

Conclusion Older age and concomitant ILD were poor prognostic factors for CTD-PCP. ST was a reasonable first-line therapy in patients with CTD-PCP, and patients with inadequate response to ST treated with atovaquone tended to have a better prognosis than those treated with pentamidine.

\section{INTRODUCTION}

Pneumocystis pneumonia (PCP) is a fatal complication that occurs in immunocompromised individuals, such as patients infected with HIV and those with connective tissue diseases (CTDs) receiving immunosuppressive therapy with glucocorticoids (GCs) and/or immunosuppressants. The reported

\section{Key messages}

What is already known about this subject?

- Pneumocystis pneumonia (PCP), an opportunistic infection commonly associated with HIV, is also observed in patients with connective tissue diseases (CTD) undergoing immunosuppressive therapy. The reported mortality rate of CTD-associated PCP (CTD-PCP) is higher than that of HIV-associated PCP (HIV-PCP).

What does this study add?

- Previous reports on CTD-PCP are limited to singlecentre observational studies and those including CTD-PCP as part of a larger cohort of non-HIV-PCP cases. The clinical course and prognostic factors of CTD-PCP remain unclear due to the lack of large studies focusing on CTD-PCP. We therefore used the diagnosis procedure combination database, a national administrative inpatients database in Japan, to address this gap in knowledge.

How might this impact on clinical practice?

- Using a large Japanese national database, this is the first study to show that CTD-PCP is a fatal complication with a mortality rate of more than $30 \%$ and that older age and concomitant interstitial lung disease are poor prognostic factors. Our analyses also reveal that sulfamethoxazole/trimethoprim is a reasonable first-line treatment option and that atovaquone might be considered as an appropriate second-line treatment option.

mortality rate of CTD-associated PCP (CTDPCP) is higher than that of HIV-associated PCP (HIV-PCP). However, no large clinical studies to date have focused on CTD-PCP, and published reports on the clinical course and prognosis of CTD-PCP have been limited to observational studies in a small number of centres and to those including CTD-PCP as part of a larger cohort of non-HIV-PCP cases, including our previous reports, because of the number of cases of CTD-PCP is smaller than that of HIV-PCP. ${ }^{1-5}$ High doses of 
sulfamethoxazole/trimethoprim (ST; trimethoprim dose, $15-20 \mathrm{mg} / \mathrm{kg} /$ day) generally comprise the firstline therapy for patients with moderate/severe PCP. ${ }^{6-8}$ However, the ST doses for PCP treatment are relatively high and often require reduction or discontinuation due to adverse effects. In patients with inadequate response to ST, second-line therapies such as atovaquone and pentamidine may be used. ${ }^{9}$ No randomised controlled trials have evaluated antibiotics in patients with CTD-PCP, and few studies have directly compared the efficacy and safety of specific antibiotics or outcomes of second-line therapeutic options such as those used in patients switching from ST. Therefore, we performed an observational study using a large, real-world database in Japan, a country with a relatively high incidence of CTD-PCP, to investigate the prognosis and prognostic factors in patients treated with specific antibiotics for CTD-PCP.

\section{METHODS}

\section{Study design and participants}

This was a retrospective cohort study using the diagnosis procedure combination (DPC) database of inpatients in Japan, which comprises all advanced treatment hospitals including all university hospitals and many acute-care community hospitals. ${ }^{10}$ Patient information recorded into the DPC database includes age, sex, diagnosis based on the $10^{\text {th }}$ revision of International Classification of Diseases and Injuries (ICD-10) codes at the time of admission, comorbidities, complications after admission and information related to treatment and procedures (eg, drug names, procedures and costs). The DPC database also contains information on the amount and duration of administered drugs, blood products used, length of hospital stay and outcomes at discharge ${ }^{11}{ }^{12}$ All data were reentered into the DPC database by a physician or professionally trained medical staff who handled the medical information. The data included in this article were provided by The DPC Study Group by permission. Data will be shared on reasonable request to the corresponding author with permission from The DPC Study Group.

Informed consent was waived for all patients included in the present retrospective cohort study, and all information extracted from the enrolled patients was anonymised.

First, 67890 patients admitted with PCP were extracted from the DPC database among 15901766 inpatient cases from 1329 hospitals registered in the DPC database between 1 April 2014 and 31 March 2016. Second, patients with CTD-PCP were defined as those who had a CTD diagnosis and were administered specific antibiotics (ST, atovaquone or pentamidine) for more than seven consecutive days to treat PCP. After the exclusion of 67890 patients with PCP in the absence of a CTD diagnosis, there were a total of 333 patients with CTD-PCP, who were included in the study (figure 1).
15,901,766 inpatients from April 1, 2014 to March 31, 2016

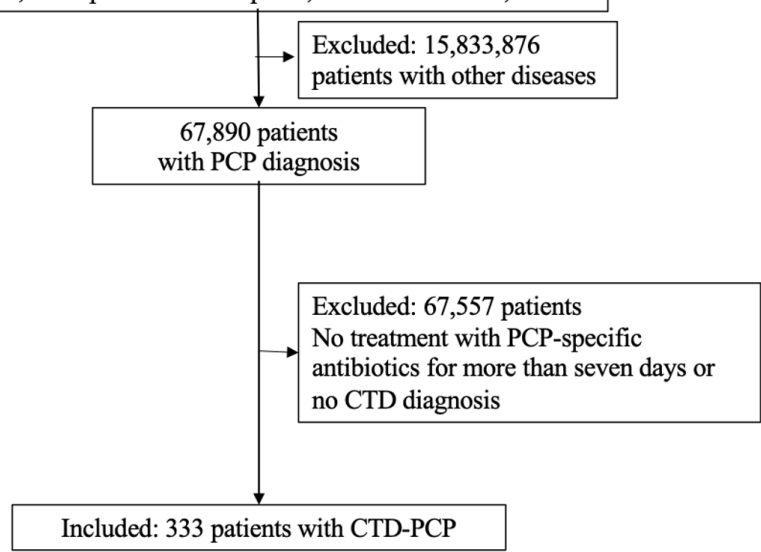

Figure 1 Flow chart of the study and analysis. CTD, connective tissue disease; PCP, pneumocystis pneumonia.

\section{Definition of CTD-PCP}

The principal diagnosis of CTD was recorded using the following ICD-10 codes: M329 (adult-onset Still's disease), M352 (Behçet's disease), M341 (CREST syndrome), M308 (cutaneous polyarteritis nodosa), M329 (dermatomyositis), M354 (eosinophilic fasciitis), M301 (eosinophilic granulomatosis with polyangiitis), M500 (Felty syndrome), M316 (giant-cell arteritis), M313 (granulomatosis with polyangiitis), M359 (IgG4related disease), M510 (Kaplan syndrome), M321 (lupus nephritis), M530 (malignant rheumatoid arthritis (RA)), M300 (microscopic polyangiitis), M351 (mixed CTD/ overlap syndrome), M300 (polyarteritis nodosa), M352 (polymyalgia rheumatica (PMR)), M332 (polymyositis), M600 (remitting seronegative symmetrical synovitis with pitting oedema), M339 (respiratory disorders due to dermatomyositis), M348 (respiratory disorders due to systemic sclerosis), M600 (RA), M510 (rheumatoid interstitial lung disease (ILD)), M520 (rheumatoid vasculitis), M349 (scleroderma), M610 (seronegative RA), M350 (Sjögren's syndrome), M329 (systemic lupus erythematosus), M340 (systemic sclerosis) and M314 (Takayasu arthritis). The principal diagnosis of PCP was based on the ICD-10 code J173.

It was expected that using the ICD-10 codes alone would identify patients who were not actually treated for PCP as well as those with suspicious PCP who initiated PCP treatment but switched to another therapeutic approach after a change in diagnosis. Therefore, based on a previous report assessing initial response to PCP treatment 7 days after the start of treatment, the present study defined patients with CTD-PCP as those with a CTD diagnosis who received one or more of the following PCP-specific antibiotics for more than seven consecutive days ${ }^{13}$ : ST (at least four tablets daily), pentamidine and atovaquone $(750 \mathrm{mg}$ two times per day). ST is generally administered at a lower dose in patients with renal dysfunction. Per the Japanese pharmaceutical reference, ST is recommended at half the usual dosage (9-12 tablets daily) in patients with renal dysfunction. Therefore, four 
tablets daily were set as the lower dose limit for PCP treatment because doses below four tablets could have been administered for prophylaxis.

\section{Study outcomes}

The primary outcome was overall in-hospital survival at 30 and 60 days after initiation of treatment for CTD-PCP. The study also aimed to investigate prognostic factors associated with in-hospital mortality with CTD-PCP and PCP-specific antibiotic treatment.

\section{Statistical analysis}

Categorical variables were presented as numbers (\%), and continuous variables were presented as medians with IQR or numbers with percentages (\%). An independent samples t-test was used to evaluate normally distributed data, and the Mann-Whitney test was employed to evaluate non-normally distributed data for comparison between two groups. Classification data number (percentage) aggregation, and the $\chi^{2}$ or Fisher's exact test. The Kaplan-Meier plots and logrank test were used to compare survival rates among groups. Associations among covariates and risk of mortality requiring dialysis were evaluated using the Cox proportional hazards regression analysis, and HRs with 95\% CIs were determined after adjusting for potential confounders. Confounding factors reported in previous studies (age, sex, and concomitant ILD) were preferentially selected as explanatory variables for multivariate analysis using the Cox proportional hazards regression. The type of CTD (RA and PMR, primary vasculitis syndrome, systemic lupus erythematosus, inflammatory myositis and systemic sclerosis) was also included in the analysis model as an explanatory variable because mortality due to PCP has been suggested to differ among different CTDs in previous studies. ${ }^{14-18}$ All tests were two-tailed, and a $\mathrm{p}<0.05$ was considered to indicate statistical significance. All statistical analyses were performed using the R software package (V.4.0.0, R Foundation). ${ }^{19}$

\section{RESULTS}

\section{Patient characteristics}

Among the 15901766 inpatients registered from 1329 hospitals between 1 April 2014 and 31 March 2016, 67890 admitted for PCP were extracted from the DPC database. Among these, 333 patients with CTD were registered as CTD-PCP for final analysis.

The baseline characteristics of the study cohort are shown in table 1 . The median age (IQR) was 71.0 (62.0, $77.0)$ years, and 210 patients $(63.1 \%)$ were women. The cohort comprised 104 (31.2\%), 109 (32.7\%) and $120(36.0 \%)$ patients who were $<65$ years, $65-74$ years (preold age) and $\geq 75$ years (old age) of age, categorised based on the Japanese Gerontological Society and the Japan Geriatrics Society. ${ }^{20}$ Additionally, 122 patients $(36.6 \%)$ had concomitant ILD. The cohort comprised $52(15.6 \%)$ and $281(84.1 \%)$ patients in the RA (including RA and/or PMR) and non-RA groups, respectively. The most common CTDs in the non-RA group were primary vasculitis $(n=116,34.8 \%)$, inflammatory myositis $(\mathrm{n}=60,18.0 \%)$ and systemic lupus erythematosus $(n=49,14.7 \%)$. Furthermore, 37 patients $(11.1 \%)$ required intensive care management and 83 patients $(24.9 \%)$ required ventilator support. GCs were administered in almost all patients $(\mathrm{n}=322,96.7 \%)$, and GC pulse therapy was given in 103 patients $(30.9 \%)$. Regarding specific antibiotics for PCP treatment, 214 patients $(64.3 \%)$ were treated with ST monotherapy.

\section{Study outcomes: survival rates and prognostic factors}

There were 114 in-hospital deaths $(34.2 \%)$ in the study cohort. The baseline characteristics of the survivor and non-survivor groups are summarised in table 2. Compared with the survivors, the non-survivors were significantly older and had significantly higher rates of vasculitis and concomitant ILD. Comparison of the treatment approaches between the two groups revealed that there were more patients receiving intensive care management and ventilator support, GC pulse therapy, pentamidineincluding second-line therapy (ie, pentamidineincluding group comprising patients treated with pentamidine monotherapy and those who switched from ST to pentamidine), immunoglobulin, albumin preparation, general antibiotics, antifungal drugs and anticytomegalovirus drugs in the non-survivor group than in the survivor group. Conversely, the rate of ST monotherapy was significantly higher in the survivor group.

The Kaplan-Meier survival curves of CTD-PCP are shown in figure 2A. The estimated survival rates at 30 and 60 days after the start of treatment for CTD-PCP were $66.0 \%$ and $53.7 \%$, respectively. We next analysed poor prognostic factors associated with in-hospital mortality due to CTD-PCP using the Cox proportional hazards regression model (table 3 ). Using the multivariable analysis, older age (HR 1.05, 95\% CI 1.03 to 1.08 , $\mathrm{p}<0.001$ ) and concomitant ILD (HR 1.65, 95\% CI 1.12 to $2.42, \mathrm{p}=0.012$ ) were poor prognostic background factors. Vasculitis, which was a significant factor in the univariable analysis, was not a poor prognostic factor in the multivariable analysis. Most patients with vasculitis were older (mean age, 74.3 vs 68.2 years in the overall cohort; $\mathrm{p}<0.001$ ), suggesting that age might be a major confounding factor. The Kaplan-Meier survival curves by the three age groups are shown in figure 2B. Briefly, the 30-day and 60-day survival rates significantly declined with increasing age $(82.4 \%$ and $76.3 \%, 65.8 \%$ and $50.1 \%$, and $53.3 \%$ and $39.9 \%$, respectively, in the $<65$ year, $65-74$ year, and $\geq 75$ year age groups, respectively). With the $<65$ year age group as reference, the HRs were 2.77 (95\% CI 1.60 to 4.90$)$ and 3.56 (95\% CI 2.03 to 6.25) for the 65-74year and $\geq 75$ year age groups, respectively $(p<0.001, \log$-rank test). The Kaplan-Meier survival curves in patients categorised according to the presence of concomitant ILD are shown in figure 2C. The 30 -day and 60-day survival rates 
RMD Open

Table 1 Patient characteristics

\begin{tabular}{|c|c|c|c|}
\hline & $\mathbf{N}$ & $\%$ & Median (IQR) \\
\hline Patients & 333 & & \\
\hline Death & 114 & & \\
\hline Age, years & & & $71(62-77)$ \\
\hline$<65$ & 104 & 31.2 & \\
\hline $65-74$ & 109 & 32.7 & \\
\hline$\geq 75$ & 120 & 36.0 & \\
\hline Female & 210 & 63.1 & \\
\hline $\mathrm{RA}$ and $\mathrm{PMR}$ & 52 & 15.6 & \\
\hline Psoriatic arthritis and spondylarthritis & 0 & 0.0 & \\
\hline Primary vasculitis syndrome & 116 & 34.8 & \\
\hline Systemic lupus erythematosus & 49 & 14.7 & \\
\hline Inflammatory myositis & 60 & 18.0 & \\
\hline Systemic sclerosis & 13 & 3.9 & \\
\hline Others & 43 & 12.9 & \\
\hline Concomitant ILD & 122 & 36.6 & \\
\hline Admission to an advanced treatment hospital & 94 & 28.2 & \\
\hline Bronchoscopy & 36 & 10.8 & \\
\hline Intensive care management & 37 & 11.1 & \\
\hline Ventilator use & 83 & 24.9 & \\
\hline Length of hospital stay after starting PCP treatment, days & & & $33(4-37)$ \\
\hline \multicolumn{4}{|l|}{ Glucocorticoid therapy } \\
\hline Concomitant glucocorticoid use & 322 & 96.7 & \\
\hline Glucocorticoid pulse therapy & 103 & 30.9 & \\
\hline \multicolumn{4}{|l|}{ Antibiotics for PCP } \\
\hline ST monotherapy & 214 & 64.3 & \\
\hline Others & 119 & 35.7 & \\
\hline PTM group & 80 & 24.0 & \\
\hline PTM monotherapy & 32 & 8.4 & \\
\hline $\mathrm{ST} \rightarrow \mathrm{PTM}$ & 48 & 14.4 & \\
\hline ATO group & 29 & 8.7 & \\
\hline ATO monotherapy & 9 & 2.7 & \\
\hline $\mathrm{ST} \rightarrow \mathrm{ATO}$ & 20 & 6.0 & \\
\hline Other combinations & 10 & 3.0 & \\
\hline \multicolumn{4}{|l|}{ Adjunctive therapy } \\
\hline Gamma-globulin & 31 & 9.3 & \\
\hline Albumin & 40 & 12.0 & \\
\hline Empiric antibiotic therapy & 245 & 73.6 & \\
\hline Antifungal drugs & 110 & 33.0 & \\
\hline Anticytomegalovirus drugs & 56 & 16.8 & \\
\hline
\end{tabular}

ATO, atovaquone; ILD, interstitial lung disease; PCP, pneumocystis pneumonia; PMR, polymyalgia rheumatica; PTM, pentamidine; RA, rheumatoid arthritis; ST, sulfamethoxazole/trimethoprim.

were significantly lower in patients with concomitant ILD (58.3\% and $47.5 \%$, respectively) than in those without concomitant ILD $(71.6 \%$ and $57.5 \%$, respectively; HR $1.60,95 \%$ CI 1.11 to $2.32 ; \mathrm{p}=0.011$, log-rank test).

\section{Treatment for CTD-PCP}

ST is generally used as the first-line antibiotic for PCP. Therefore, we primarily compared survival rates between the patients who were treated with ST monotherapy and those who were treated with antibiotics other than ST or a combination of ST and other antibiotics (others group) to evaluate the efficacy of first-line treatment with ST for CTD-PCP. There were no significant differences in patient background characteristics between the ST monotherapy and others groups (table 4). The comparison of survival 
Table 2 Comparison of the clinical features between the survivors and non-survivors

\begin{tabular}{|c|c|c|c|c|c|}
\hline & \multirow{2}{*}{$\begin{array}{l}\text { Survivors } \\
\mathrm{N}=219\end{array}$} & \multirow[b]{2}{*}{$\%$} & \multirow{2}{*}{$\begin{array}{l}\text { Non-survivors } \\
\mathrm{N}=114\end{array}$} & \multirow[b]{2}{*}{$\%$} & \multirow[b]{2}{*}{$P$ value } \\
\hline & & & & & \\
\hline Age, years (median, IQR) & $65.3(57-76)$ & & $73.6(68-81)$ & & $<0.001$ \\
\hline Female & 146 & 66.7 & 64 & 56.1 & 0.073 \\
\hline RA and PMR & 37 & 16.9 & 15 & 13.2 & 0.428 \\
\hline Non-RA and PMR & 182 & 83.1 & 99 & 86.8 & \\
\hline Primary vasculitis syndrome & 63 & 28.8 & 53 & 46.5 & 0.002 \\
\hline Systemic lupus erythematosus & 32 & 14.6 & 17 & 14.9 & 1.000 \\
\hline Inflammatory myositis & 45 & 20.5 & 15 & 13.2 & 0.101 \\
\hline Systemic sclerosis & 10 & 4.7 & 3 & 2.6 & 0.554 \\
\hline Others & 32 & 14.6 & 11 & 9.6 & 0.231 \\
\hline Concomitant ILD & 70 & 32.0 & 52 & 45.6 & 0.017 \\
\hline Admission to an advanced treatment hospital & 66 & 30.1 & 28 & 24.6 & 0.284 \\
\hline Bronchoscopy & 25 & 11.4 & 11 & 9.6 & 0.712 \\
\hline Intensive care management & 12 & 5.5 & 25 & 21.9 & $<0.001$ \\
\hline Ventilator use & 16 & 7.3 & 67 & 58.8 & $<0.001$ \\
\hline Length of hospital stay after starting PCP treatment & $35.8(6-24)$ & & $27.3(4-18)$ & & 0.034 \\
\hline Concomitant glucocorticoid use & 210 & 95.9 & 112 & 98.2 & 0.343 \\
\hline Glucocorticoid pulse therapy & 46 & 21.0 & 57 & 50.0 & $<0.001$ \\
\hline ST monotherapy & 154 & 70.3 & 60 & 52.6 & 0.017 \\
\hline Others & 65 & 29.7 & 54 & 47.4 & \\
\hline PTM monotherapy or ST $\rightarrow$ PTM (PTM group) & 34 & 15.5 & 46 & 40.4 & $<0.001$ \\
\hline ATO monotherapy or ST $\rightarrow$ ATO (ATO group) & 24 & 10.9 & 5 & 4.4 & 0.063 \\
\hline Other combinations & 7 & 3.3 & 3 & 2.6 & 1.000 \\
\hline
\end{tabular}

t-test, the Mann-Whitney test, $\chi^{2}$ test and Fisher's exact test were used when appropriate to compare the groups.

ATO, atovaquone; ILD, interstitial lung disease; PCP, pneumocystis pneumonia; PMR, polymyalgia rheumatica; PTM, pentamidine; RA, rheumatoid arthritis; ST, sulfamethoxazole/trimethoprim.

rates between the ST monotherapy and other groups is shown in figure 2D. Briefly, both the 30-day and 60-day survival rates were significantly higher in the ST monotherapy group than in the others group (30-day and 60-day survival rates, ST monotherapy group, $79.0 \%$ and $76.6 \%$ versus others group, $63.0 \%$ and $58.5 \%$, respectively; HR $0.64,95 \%$ CI 0.44 to $0.82, p=0.015, \log$-rank test). Pentamidine or atovaquone was generally used in patients with inadequate response to ST.

The patients who were treated with pentamidine $(n=32)$ and those who were switched from ST to pentamidine $(\mathrm{n}=48)$ were categorised as the pentamidine group $(n=80)$, whereas the patients who were treated with atovaquone $(n=9)$ monotherapy and those who were switched from ST to atovaquone $(n=20)$ were categorised as the atovaquone group $(n=29)$. Although there were no significant differences in patient background characteristics between pentamidine and atovaquone groups (table 4), both the 30-day and 60-day survival rates were significantly higher in the atovaquone group than in the pentamidine group (30-day and 60-day survival rates, atovaquone group, $82.2 \%$ and $82.2 \%$ vs pentamidine group, $49.0 \%$ and $37.7 \%$, respectively; HR $3.06,95 \%$ CI 1.22 to $7.73, \mathrm{p}=0.018$, log-rank test) (figure $2 \mathrm{E}$ ).

\section{DISCUSSION}

The present observational study including 333 patients with CTD-PCP using the DPC database, a Japanese nationwide database, revealed that CTD-PCP remained a fatal complication and that older age and concomitant ILD were background factors associated with poor prognostic. Among patients with inadequate response to ST, the prognosis was better in those who received atovaquone compared with pentamidine as second-line treatment. The results of the present nationwide study with a larger cohort of patients provide stronger support to previous reports showing older age and concomitant ILD as risk and poor prognostic factors for PCP. ${ }^{14-18}$

Because of the relatively good prognosis of patients treated with ST alone, ST might be considered as a reasonable first-line antibiotic for CTD-PCP as well as for HIV-PCP. However, patients treated with ST monotherapy might have been less likely to have complications such as chronic kidney disease, which is a prognostic factor 


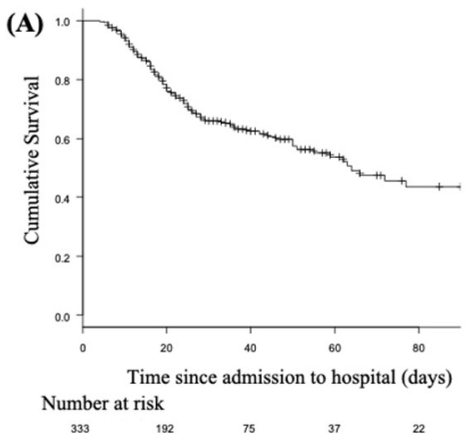

(B)

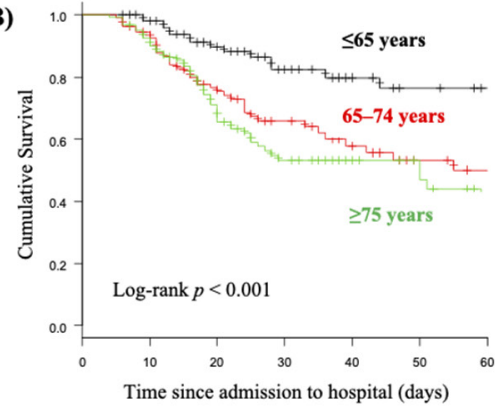

Number at risk
(C)

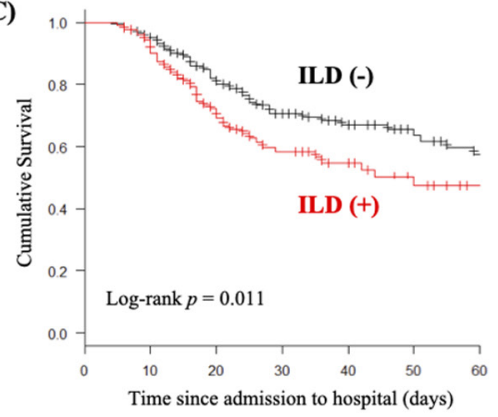

Number at risk
(D)

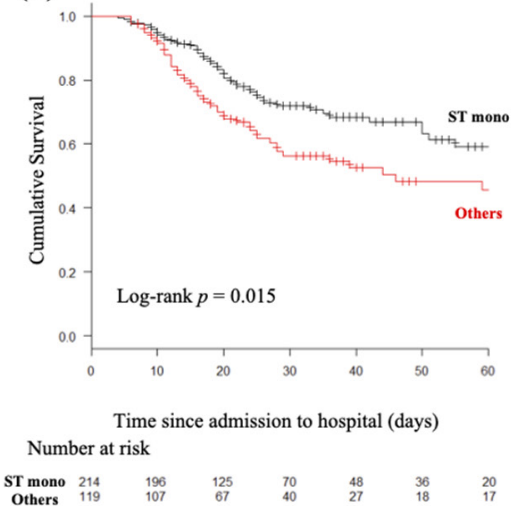

(E)

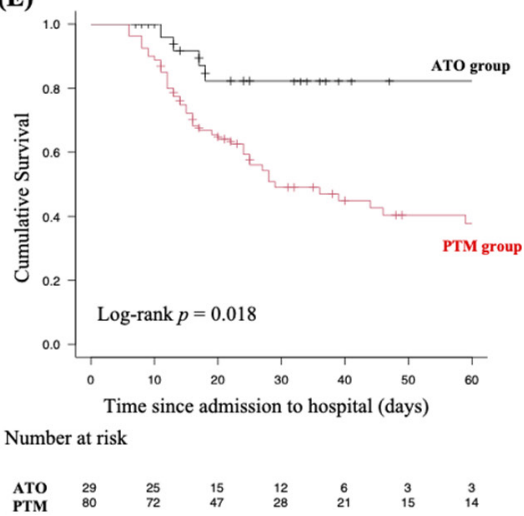

Figure 2 (A) Estimated Kaplan-Meier overall survival curve of patients with connective tissue disease-associated pneumocystis pneumonia (CTD-PCP). (B) Estimated Kaplan-Meier overall survival curves of patients with CTD-PCP by age category. (C) Estimated Kaplan-Meier overall survival curves of patients with CTD-PCP with or without interstitial lung disease (ILD). (D) Estimated Kaplan-Meier overall survival curves of patients with CTD-PCP who completed sulfamethoxazole/ trimethoprim (ST) monotherapy (mono). (E) Estimated Kaplan-Meier overall survival curves of patients with CTD-PCP who were treated with second-line therapy (pentamidine (PTM) or atovaquone (ATO)).

for PCP and should be considered as a confounding factor. ${ }^{21}$ The present study results suggest that the prognosis might be relatively poor in patients treated with pentamidine-including regimens. Since low-dose ST with $4-10 \mathrm{mg} / \mathrm{kg}$ /day trimethoprim has also been suggested to be as effective as high-dose ST in patients with nonHIV-PCP, a reduction in ST dose might be considered as a next step in patients with inadequate response to $\mathrm{ST}^{22}$ There are several potential reasons that might explain the poor prognosis observed in the pentamidine group. Pentamidine is administered intravenously and might have been used in patients with relatively poor general condition. In addition, pentamidine has a relatively high rate of side adverse effects such as renal dysfunction, cytopenia and abnormal glucose tolerance and it cannot be denied that patients treated with pentamidine might not have been treated with a sufficient dose for an adequate length of time. Studies suggest that pentamidine as firstline or second-line treatment is associated with high risk of death and may require a change in treatment in patients with HIV-PCP. ${ }^{23}$ Therefore, pentamidine should be used in carefully selected individuals, such as those with severe disease exhibiting inadequate response to ST and/or atovaquone. 
Table 3 Prognostic factors for survival

\begin{tabular}{|c|c|c|c|c|c|c|c|}
\hline \multirow[b]{2}{*}{ Variable } & \multirow[b]{2}{*}{$\mathbf{N}$} & \multicolumn{3}{|c|}{ Univariable Cox hazard analysis } & \multicolumn{3}{|c|}{ Multivariable Cox hazard analysis } \\
\hline & & HR & $95 \% \mathrm{Cl}$ & $P$ value & HR & $95 \% \mathrm{Cl}$ & $P$ value \\
\hline Age & & 1.05 & 1.03 to 1.07 & $<0.001$ & 1.06 & 1.03 to 1.08 & $<0.001$ \\
\hline Male & 128 & Ref & & & Ref & & \\
\hline Female & 205 & 0.62 & 0.43 to 0.90 & 0.012 & 0.75 & 0.51 to 1.11 & 0.158 \\
\hline Concomitant ILD & 122 & 1.60 & 1.11 to 2.32 & 0.012 & 1.65 & 1.12 to 2.42 & 0.012 \\
\hline RA and PMR & 52 & 0.97 & 0.56 to 1.67 & 0.901 & 0.98 & 0.44 to 2.20 & 0.967 \\
\hline Primary vasculitis syndrome & 116 & 1.63 & 1.12 to 2.36 & 0.010 & 1.14 & 0.57 to 2.26 & 0.711 \\
\hline Systemic lupus erythematosus & 49 & 0.87 & 0.51 to 1.46 & 0.589 & 2.07 & 0.94 to 4.58 & 0.071 \\
\hline Inflammatory myositis & 60 & 0.69 & 0.40 to 1.18 & 0.177 & 1.15 & 0.52 to 2.54 & 0.722 \\
\hline Systemic sclerosis & 13 & 0.71 & 0.22 to 2.23 & 0.552 & 0.81 & 0.22 to 2.96 & 0.746 \\
\hline Others & 43 & 0.82 & 0.21 to 3.23 & 0.775 & & & \\
\hline Oxygen administration on admission & 76 & 0.84 & 0.52 to 1.35 & 0.465 & & & \\
\hline
\end{tabular}

Univariable and multivariable Cox proportional hazards regression analyses were performed.

ILD, interstitial lung disease; PMR, polymyalgia rheumatica; RA, rheumatoid arthritis; Ref, reference.

Atovaquone has been reported to be a relatively welltolerated antibiotic for PCP. ${ }^{25}$ In the present study, the 30-day and 60-day survival rates were comparable between the ST monotherapy $(79.0 \%$ and $76.6 \%$, respectively) and atovaquone ( $72.2 \%$ and $72.2 \%$, respectively) groups. It is possible that, as an oral antibiotic, atovaquone might have been administered to patients in relatively good general condition in the current study. On the basis of the results, the efficacy of atovaquone may be comparable with that of ST for mild to moderate CTDPCP cases that can be treated orally. Future studies with a larger number of cases are warranted for the assessment of patient background characteristics such as severity of pneumonia and renal dysfunction to further elucidate the role of atovaquone in outcomes of patients with
CTD-PCP. This study was conducted using the DPC data encompassing a 2-year period starting in April 2014. The limited number of patients treated with atovaquone was due to its recent approval for use in Japan in 2012; atovaquone might not have been widely used for the treatment of PCP during the observation period. Nonetheless, the present study findings show for the first time that ST was a reasonable first-line treatment for CTD-PCP and that the prognosis with atovaquone was better than that with pentamidine in patients receiving second-line antibiotic treatment following inadequate response to ST. 'Consensus statements for medical practice: Biological agents and lung diseases,' which includes a description of PCP, was published in 2014 in Japan. ${ }^{26}$ Although PCP has been described in patients using biologics, information

Table 4 Comparison of the clinical features by treatment group

\begin{tabular}{|c|c|c|c|c|c|c|c|c|c|c|}
\hline & ST monotherapy & & Other & & & PTM & & ATO & & \\
\hline & $\mathrm{N}=214$ & $\%$ & $\mathrm{~N}=119$ & $\%$ & $P$ value & $\mathrm{N}=80$ & $\%$ & $\mathrm{~N}=\mathbf{2 9}$ & $\%$ & $P$ value \\
\hline Age (median, IQR) & $71.0(60.0-77.0)$ & & $71.0(63.0-77.5)$ & & 0.640 & $71(65-76)$ & & $73(59-81)$ & & 0.942 \\
\hline RA and PMR & 30 & 14.0 & 22 & 18.5 & 0.345 & 13 & 16.3 & 7 & 24.1 & 0.403 \\
\hline Non-RA and PMR & 184 & 86.0 & 97 & 81.5 & & 67 & 83.8 & 22 & 75.9 & \\
\hline $\begin{array}{l}\text { Systemic lupus } \\
\text { erythematosus }\end{array}$ & 26 & 12.1 & 23 & 19.3 & 0.106 & 17 & 21.3 & 3 & 10.3 & 0.267 \\
\hline Inflammatory myositis & 36 & 16.8 & 24 & 20.2 & 0.460 & 14 & 17.5 & 7 & 24.1 & 0.425 \\
\hline Systemic sclerosis & 9 & 4.2 & 4 & 3.4 & 0.777 & 1 & 1.3 & 2 & 6.9 & 0.172 \\
\hline Others & 31 & 14.5 & 12 & 10.1 & 0.307 & 10 & 12.5 & 2 & 6.9 & 0.510 \\
\hline
\end{tabular}

t-test, the Mann-Whitney test, $\chi^{2}$ test and Fisher's exact test were used when appropriate to compare the groups.

ATO, atovaquone; ILD, interstitial lung disease; PMR, polymyalgia rheumatica; PTM, pentamidine; RA, rheumatoid arthritis. 
is limited on treatment recommendations for patients using immunosuppressive drugs other than biologics and for second-line PCP treatments (atovaquone or pentamidine). Moreover, evidence for CTD-PCP is still insufficient. We used the DPC database to emphasise the coverage of cases, considering that CTD-PCP is a rare disease, and presented the treatment status and prognosis in this study. Owing to the limitation of having a small number of patients, we could not sufficiently adjust for patient background and confounding factors in each treatment group. Therefore, we must be careful in interpreting the results on survival rates; however, we would like to base the results of this study on clinical research in a future study to evaluate the effects of drug treatments.

Our study has several limitations due to the retrospective, non-randomised, observational study design. Despite the use of a nationwide database, the study cohort comprised only 333 patients. Therefore, it was difficult to adequately adjust for previously reported confounding factors in the multivariable analysis. The GC dose and type and dose of immunosuppressants, with or without prophylaxis administered at the time of PCP onset, have also been proposed as potential prognostic factors for evaluating antibiotic treatment in patients with CTDPCP and should therefore be included as confounding factors. ${ }^{27}$ However, the DPC database does not contain this information and hence could not be included in the adjustment of patient background characteristics. ${ }^{28} 29$ One approach to overcome this limitation would be to adjust the background characteristics after matching the DPC data with the National Database of Health Insurance Claims data. The DPC database, which contains medical information during hospitalisation, and the National Database of Health Insurance Claims database, which contains medical information, such as prescription drugs in outpatient settings, were treated as separate databases. As there are many institutional, technical and ethical issues in the identification of individuals and linking of both databases, we could not link the databases from which we sampled the data in this study from 2014 to 2016. If future institutional changes and technological innovations permit us to link the databases before and during hospitalisation, we would like to investigate the linking of individuals' data.

GCs are also used as treatments for moderate to severe PCP. $^{30}{ }^{31}$ Although the administration of GCs may affect the prognosis of CTD-PCP, it was difficult to accurately identify the dosage, duration and purposes of GCs due to the limitations of the DPC database. Therefore, to avoid misleading the readers we restricted our description to whether GCs were administered or not. To resolve this limitation, we consider combining a medical record review in a future study.

Additionally, the DPC database cannot track disease course after hospital discharge or transfer of the patient to another institution. Therefore, the outcomes of patients after their transfer to hospitals other than those participating in the DPC database or after their admittance to a non-DPC-participating hospital after discharge are not known. Laboratory results, microbiological test results, imaging findings and medical records are not available in the DPC database. ${ }^{32}$ The disease diagnoses, including comorbidities, and causes of death were based on the data extracted from the DPC database, and the diagnostic basis was not completely supported. Previous studies on PCP using the administrative or claims database diagnosed and defined PCP using ICD codes. ${ }^{3334} \mathrm{On}$ the basis of these reports, we also made a diagnosis and definition based on the ICD-10 codes. We also defined them according to the antibiotics administered for PCP during hospitalisation to ensure the validity of the diagnosis. Antibiotics for treating PCP, such as high-dose ST, atovaquone and pentamidine, are unlikely to be used for infections other than PCP. Cases in which antibiotics for treating PCP have been administered for more than a certain period are considered to have a high probability of true PCP. It is reasonable to assume that patients with CTD-PCP were likely to be administered immunosuppressive therapy, because PCP occurs in immunocompromised hosts. Therefore, patients with CTD-PCP are more likely to have a definitive diagnosis of CTD. Regarding the diagnosis recorded in the DPC database, they are entered by the physician in charge of the treatment or by a specially trained medical professional under the supervision of a physician. The validity of DPC database has been reported to be moderate (eg, $68.8 \%$ sensitivity, $97.5 \%$ specificity, $75.9 \%$ positive predictive value and $96.5 \%$ negative predictive value for congestive heart failure) ${ }^{35}$ In relatively rare diseases, such as CTD-PCP, research has been insufficient to evaluate the validity. Thus, further investigation is needed in the future.

In the present nationwide study using the DPC database, we found that CTD-PCP remained a fatal complication with a mortality rate of more than $30 \%$ and that older age and concomitant ILD were poor prognostic factors. ST might be a reasonable first-line antibiotic in CTD-PCP as well as in HIV-PCP. In patients with inadequate response to ST, if available, treatment with atovaquone might achieve good prognosis comparable to that of ST.

Contributors YI, KN and KT conceptualised and designed the study and provided substantial contributions to analysis and interpretation of data. KT and HM made contributions to data collection. All authors contributed to drafting of the article and approved the final version of the article for publication. YF, SM and YT supervised the interpretation of data and manuscript.

Funding The authors have not declared a specific grant for this research from any funding agency in the public, commercial or not-for-profit sectors.

Competing interests YT has received speaking fees and/or honoraria from Daiichi-Sankyo, Astellas, Chugai, Eli Lilly, Pfizer, Abbvie, YL Biologics, Bristol-Myers, Takeda, Mitsubishi-Tanabe, Novartis, Eisai, Janssen, and Teijin and has received research grants from Asahi-kasei, Mitsubishi-Tanabe, Chugai, Takeda, Sanofi, Bristol-Myers, UCB, Daiichi-Sankyo, Eisai, and Ono. KN has received research grants from Mitsubishi-Tanabe, Eli Lilly, and Eisai.

Patient consent for publication Not required.

Ethics approval The study protocol was approved by The Ethics Committee of Medical Care and Research of the University of Occupational and Environmental Health, Japan (approval number: R2-007). 
Provenance and peer review Not commissioned; externally peer reviewed.

Data availability statement Data are obtained from the diagnosis procedure combination (DPC) database of inpatients in Japan and are not publicly available. Data are available upon reasonable request.

Open access This is an open access article distributed in accordance with the Creative Commons Attribution Non Commercial (CC BY-NC 4.0) license, which permits others to distribute, remix, adapt, build upon this work non-commercially, and license their derivative works on different terms, provided the original work is properly cited, appropriate credit is given, any changes made indicated, and the use is non-commercial. See: http://creativecommons.org/licenses/by-nc/4.0/.

ORCID iDs

Yuichi Ishikawa http://orcid.org/0000-0001-7406-2309

Yoshiya Tanaka http://orcid.org/0000-0002-0807-7139

\section{REFERENCES}

1 Thomas CF, Limper AH. Pneumocystis pneumonia. N Engl J Med 2004;350:2487-98.

2 Mori S, Sugimoto M. Pneumocystis jirovecii infection: an emerging threat to patients with rheumatoid arthritis. Rheumatology 2012;51:2120-30.

3 Falagas ME, Manta KG, Betsi Gl, et al. Infection-Related morbidity and mortality in patients with connective tissue diseases: a systematic review. Clin Rheumatol 2007;26:663-70.

4 Katsuyama T, Saito K, Kubo S, et al. Prophylaxis for Pneumocystis pneumonia in patients with rheumatoid arthritis treated with biologics, based on risk factors found in a retrospective study. Arthritis Res Ther 2014;16:R43.

5 Saito K, Nakayamada S, Nakano K, et al. Detection of Pneumocystis carinii by DNA amplification in patients with connective tissue diseases: re-evaluation of clinical features of P. carinii pneumonia in rheumatic diseases. Rheumatology 2004:43:479-85.

6 Winston DJ, Lau WK, Gale RP, et al. Trimethoprim-Sulfamethoxazole for the treatment of Pneumocystis carinii pneumonia. Ann Intern Med 1980;92:762-9.

7 Safrin S, Finkelstein DM, Feinberg J, et al. Comparison of three regimens for treatment of mild to moderate Pneumocystis carinii pneumonia in patients with AIDS. A double-blind, randomized, trial of oral trimethoprim-sulfamethoxazole, dapsone-trimethoprim, and clindamycin-primaquine. ACTG 108 Study Group. Ann Intern Med 1996;124:792-802.

8 Hughes W, Leoung G, Kramer F, et al. Comparison of atovaquone (566C80) with trimethoprim-sulfamethoxazole to treat Pneumocystis carinii pneumonia in patients with AIDS. N Engl J Med 1993;328:1521-7.

9 Salzer HJF, Schäfer G, Hoenigl M, et al. Clinical, diagnostic, and treatment disparities between HIV-infected and non-HIV-infected immunocompromised patients with Pneumocystis jirovecii pneumonia. Respiration 2018;96:52-65.

10 Matsuda S. Development of case mix based evaluation system in Japan. Jpn Hosp 2016;35:35-44.

11 Isogai $\mathrm{T}$, Yasunaga $\mathrm{H}$, Matsui $\mathrm{H}$, et al. Factors affecting in-hospital mortality and likelihood of undergoing surgical resection in patients with primary cardiac tumors. J Cardiol 2017;69:287-92.

12 Iwagami M, Yasunaga H, Doi K, et al. Postoperative polymyxin $B$ hemoperfusion and mortality in patients with abdominal septic shock: a propensity-matched analysis. Crit Care Med 2014;42:1187-93.

13 Benfield T, Atzori C, Miller RF, et al. Second-Line salvage treatment of AIDS-associated Pneumocystis jirovecii pneumonia: a case series and systematic review. J Acquir Immune Defic Syndr 2008;48:63-7.

14 Kotani T, Katayama S, Miyazaki Y, et al. Risk Factors for the Mortality of Pneumocystis jirovecii Pneumonia in Non-HIV Patients Who Required Mechanical Ventilation: A Retrospective Case Series Study. Biomed Res Int 2017;2017:1-7.

15 likuni N, Kitahama M, Ohta S, et al. Evaluation of Pneumocystis pneumonia infection risk factors in patients with connective tissue disease. Mod Rheumatol 2006;16:282-8.

16 Watanabe K, Sakai R, Koike R, et al. Clinical characteristics and risk factors for Pneumocystis jirovecii pneumonia in patients with rheumatoid arthritis receiving adalimumab: a retrospective review and case-control study of 17 patients. Mod Rheumatol 2013;23:1085-93.

17 Yoshida Y, Takahashi Y, Minemura N, et al. Prognosis of Pneumocystis pneumonia complicated in patients with rheumatoid arthritis $(\mathrm{rA})$ and non-RA rheumatic diseases. Mod Rheumatol 2012;22:509-14.

18 Kageyama T, Furuta S, Ikeda K, et al. Prognostic factors of Pneumocystis pneumonia in patients with systemic autoimmune diseases. PLoS One 2019;14:e0214324.

19 Kanda Y. Investigation of the freely available easy-to-use software 'EZR' for medical statistics. Bone Marrow Transplant 2013;48:452-8.

20 Ouchi Y, Rakugi $\mathrm{H}$, Arai $\mathrm{H}$, et al. Redefining the elderly as aged 75 years and older: Proposal from the Joint Committee of Japan Gerontological Society and the Japan Geriatrics Society. Geriatr Gerontol Int 2017;17:1045-7.

21 Minowa K, Nakiri Y, Lee S, et al. [Examination of availability of the criteria for protective therapy against Pneumocystis pneumonia]. Nihon Rinsho Meneki Gakkai Kaishi 2009;32:256-62.

22 Nakashima K, Aoshima M, Nakashita T, et al. Low-Dose trimethoprim-sulfamethoxazole treatment for Pneumocystis pneumonia in non-human immunodeficiency virus-infected immunocompromised patients: a single-center retrospective observational cohort study. J Microbiol Immunol Infect 2018:51:810-20.

23 Helweg-Larsen J, Benfield T, Atzori C, et al. Clinical efficacy of first- and second-line treatments for HIV-associated Pneumocystis jirovecii pneumonia: a tri-centre cohort study. J Antimicrob Chemother 2009;64:1282-90.

24 El-Sadr WM, Murphy RL, Yurik TM, et al. Atovaquone compared with dapsone for the prevention of Pneumocystis carinii pneumonia in patients with HIV infection who cannot tolerate trimethoprim, sulfonamides, or both. Community Program for Clinical Research on AIDS and the AIDS Clinical Trials Group. N Engl J Med 1998;339:1889-95.

25 Dohn MN, Weinberg WG, Torres RA, et al. Oral atovaquone compared with intravenous pentamidine for Pneumocystis carinii pneumonia in patients with AIDS. atovaquone Study Group. Ann Intern Med 1994;121:174-80.

26 Tokuda $\mathrm{H}$, Harigai $\mathrm{M}$, Kameda $\mathrm{H}$, et al. Consensus statements for medical practice: Biological agents and lung disease [Abridged English translation by the Japanese Respiratory Society]. Respir Investig 2017;55:229-51.

27 Winthrop KL, Baddley JW. Pneumocystis and glucocorticoid use: to prophylax or not to prophylax (and when?); that is the question. Ann Rheum Dis 2018;77:631-3.

28 Mecoli CA, Saylor D, Gelber AC, et al. Pneumocystis jiroveci pneumonia in rheumatic disease: a 20-year single-centre experience. Clin Exp Rheumatol 2017;35:671-3.

29 Zhang Y, Zheng Y. Pneumocystis jirovecii pneumonia in mycophenolate mofetil-treated patients with connective tissue disease: analysis of 17 cases. Rheumatol Int 2014;34:1765-71.

30 Ewald H, Raatz H, Boscacci R, et al. Adjunctive corticosteroids for Pneumocystis jiroveci pneumonia in patients with HIV infection. Cochrane Database Syst Rev 2015;4:CD006150.

31 Ding L, Huang $\mathrm{H}$, Wang $\mathrm{H}$, et al. Adjunctive corticosteroids may be associated with better outcome for non-HIV Pneumocystis pneumonia with respiratory failure: a systemic review and metaanalysis of observational studies. Ann Intensive Care 2020;10:34.

32 Hayashida K, Murakami G, Matsuda S, et al. History and profile of diagnosis procedure combination (DPC): development of a real data collection system for acute inpatient care in Japan. $J$ Epidemiol 2021;31:1-11.

33 Murray SG, Schmajuk G, Trupin L, et al. National lupus hospitalization trends reveal rising rates of herpes zoster and declines in Pneumocystis pneumonia. PLoS One 2016;11:e0144918.

34 Ward MM, Donald F. Pneumocystis carinii pneumonia in patients with connective tissue diseases: the role of hospital experience in diagnosis and mortality. Arthritis Rheum 1999;42:780-9.

35 Yamana $\mathrm{H}$, Moriwaki $\mathrm{M}$, Horiguchi $\mathrm{H}$, et al. Validity of diagnoses, procedures, and laboratory data in Japanese administrative data. $J$ Epidemiol 2017;27:476-82. 